\title{
RISOLUZIONE DI UNA CLASSE DI EQUAZIONI FUNZIONALI.
}

\author{
Nota di S. Pincherle, in Bologna.
}

Adunanza del 22 maggio 1904

Le equazioni alle differenze e le equazioni funzionali analoghe, in quanto possono servire alla determinazione di funzioni analitiche, non sono state molto studiate. Prescindendo dai lavori anteriori all'epoca delle odierne vedute nella teoria delle funzioni, rimangono quasi isolati il lavoro del Guichard *) sull'integrazione finita di una funzione intera e la bella memoria dell'Hurwitz ${ }^{* *}$ ) cui esso ha dato origine; e lo stesso si può dire delle interessanti pubblicazioni del Mesurn sparse nella raccolta degli "Acta Mathematica », e di alcuni miei lavori ***).

Eppure simili equazioni offrono molto interesse per sè, e per le applicazioni cui esse dànno luogo: esse si presentano nello studio delle singolariti delle funzioni definite da uno sviluppo di TAYLOR, in quelle delle trascendenti intere, ecc.; inoltre si trova campo di applicare in modo assar naturale, nella loro risoluzione, alcuni dei sussidì più recenti dell'Analisi, come la teoria delle serie assintotiche del PorNCARÉ ${ }^{* * *}$ ) ed

*) Annales de l'École Normale Supérieure, III série, t. IV, pag. 36r (1887).

**) Actr Mathematica, t. XX, pag 285.

***) Per esempio le due memorie:

Sulla isoluzione dell'equazione $\sum h_{\nu} \varphi\left(x+a_{\nu}\right)=f(x)$, a coeffictentz costanti, e Sulla risoluzione dell'equazione $\sum h_{\nu} \varphi\left(x+a_{\nu}\right)=f(x)$, a coefficienti razionali [Memorie dell'Accademia di Bologna, s. IV, t IX (I888)]. - Cfr. Halphen, Comptes Rendus de l'Académie des Sciences, t. XCIII, pag. 78I (I88I).

****) V. più avanti, il $\$ 27$ di questo lavoro. 
anche, come ha mostrato recentemente il FÉJER *), la teoria della sommabilità secondo il Borel. Pur nonostante, non sembra che sia stato fatto fino ad ora uno studio sistematico di classi generali di simili equazioni.

La presente Nota si propone di portare un contributo a codesto studio: in essa si esporrà un metodo per la risoluzione generale dell'equazione

$$
A(b)=a,
$$

dove $a$ è una funzione nota, $b$ una funzione incognita, ed $A$ un'operazione che si supporrà commutabile colla derivazione. È evidente che l'equazione considerata contiene, come casi particolarissimi, le equazioni differenziali o alle differenze finite lineari e a coefficienti costanti. Il problema verrà risoluto in due casi, che si distinguono per l'ipotesi che si fara circa all'insieme funzionale cui appartengono la funzione data $a$ e la funzione incognita $b$.

Conviene aggiungere che il metodo qui indicato per la risoluzione dell'equazione (a) si estende, con lievi modificazioni, alla soluzione dell'equazione più generale

$$
\alpha(x) A(b)+\alpha_{\mathrm{r}}(x) A_{\mathrm{r}}(b)+\cdots \alpha_{p}(x) A_{p}(b)=a,
$$

dove $\alpha, \alpha_{1}, \ldots \alpha_{p}$ sono funzioni razionali, $A, A_{1}, \ldots A_{p}$ sono operazioni commutabili con $D$, $a$ è la funzione data e $b$ la funzione incognita.

\section{I. - Notazioni e richiami.}

I. In ciò che segue, indicheremo costantemente le operazioni distributive mediante le lettere maiuscole dell'alfabeto romano. In particolare, $D$ rappresenterd l'operazione di derivazione.

2. Le funzioni o elementi di funzioni verranno rappresentate colle lettere minuscole dell'alfabeto greco. Però, le funzioni determinanti, definite al $\ 4$, verranno indicate colle lettere $a, b, c, \ldots$

3. L'insieme (o spazio funzionale) dei rami di funzioni analitiche della variabile $x$ regolari in un intorno di $x=0$, si indicherà col simbolo $S$. La somma di quanti si vogliano elementi di $S$ dà un elemento

*) Comptes Rendus de l'Académie des Sciences, t. CXXXVII, 23 nov. rgo3. 
dello spazio medesimo: ciù si esprime dicendo che lo spazio $S$ è lineare. Entro lo spazio $S$ si possono distinguere infiniti spazi, pure lineari; in particolare, si può considerare lo spazio, che si indicherà con $S_{r}$, di tutte le serie di potenze convergenti entro un cerchio di centro $x=0$ e di raggio maggiore di $r$. Dei due spazî $S_{r}, S_{t}$, dove si suppone $r>t$, il primo è contenuto nel secondo. In particolare, $S_{\mathrm{o}}$ è l'insieme di tutte le serie di potenze aventi un raggio non nullo di convergenza, e non differisce da $S ; S_{\infty}$ è l'insieme di tutre le funzioni intere e lo indıcheremo con $E$; esso è contenuto in ogni $S_{r}$. Alla sua volta, $E$ contiene infiniti spazi lneari; fra questi, indicheremo con $E_{r}$ l'nsieme delle funzioni intere $\sum c_{n} x^{n}$ tall che $\sum n ! c_{n} x^{n}$ appartenga ad $S_{r}$.

4. Daremo il nome di funzione determinante ad ogni funzione $\boldsymbol{a}(\boldsymbol{x})$ di $x$ rappresentabile sotto la forma

$$
a(x)=\int_{0}^{\infty} e^{-t x} \varphi(t) d t
$$

$\mathrm{Ci}$ limiteremo al caso in cui $\varphi(t)$, oltre ad essere finita ed atta all'integrazione fra o ed $\infty$, sia anche continua ed ammetta le derivate di tutti gli ordıni, pure finite e continue nello stesso intervallo. E noto, dalla teoria delle funzioni rappresentabili nella forma (I), che se l'integrale ha un significato per $x=x_{0}$, esso avrà senso per ogni $x$ tale che sia

$$
\left.R(x)>R\left(x_{0}\right)^{*}\right)
$$

si sa pure che nel semi-piano definito da (2), la $a(x)$ è analitica regolare e che essa tende a zero se $x$ tende all'infinito nella direzione dell'asse reale positivo. Si sa di più $\left.{ }^{* *}\right)$ che la funzione $\varphi(t)$, che è detta fnnzione generatruce di $a(x)$, è univocamente determinata dalla (I), e che essa funzione è espressa, mediante la sua funzione determinante, dall'integrale definito:

$$
\varphi(t)=\frac{\mathrm{I}}{2 \pi i} \int_{k-i \infty}^{k+i \infty} e^{t x} \boldsymbol{a}(x) d x,
$$

dove $t$ è reale e positivo, e $k$ è un numero reale arbitrario, purchè superiore ad $R\left(x_{0}\right)$.

*) Con $R(x)$ si intende «la parte reale di $x$ ».

**) V. Lerch, Acta Mathematica, t. XXVII, pag. 347. 
5. Le relazioni (I) e (3), fra loro inverse, definiscono una trasformazione funzionale cui dal PoINcaré, che ne ha studiato notevoli applicazioni, è stato dato il nome trasformazione di LAPLACE. Per brevità, le relazioni (I) e (3) si possono rappresentare mediante simboli operatorî, ponendo

(4)

$$
\boldsymbol{a}(x)=J[\varphi(t)], \quad \varphi(t)=G[\boldsymbol{a}(x)] .
$$

Fra le proprietà della trasformazione di LAPLACE ricorderemo le seguenti, di cui dovremo fare uso. Derivando la (I) si ottiene

$$
\text { (5) } \quad \int_{0}^{\infty} e^{-x t} t^{n} \varphi(t) d t=(-\mathrm{I})^{n} D^{n} a(x), \quad(n=\mathrm{I}, 2,3, \ldots)
$$

che si può scrivere simbolicamente

$$
J\left[t^{n} \varphi(t)\right]=(-\mathrm{I})^{n} D^{n} J \varphi .
$$

Integrando per parti la medesima (I), viene

$$
x J \varphi=J D \varphi+\varphi(0) \text {. }
$$

6. L'insieme delle funzioni determinanti costituisce uno spazio lineare, che indicheremo con $D$. A questo spazio appartengono, in particolare, tutti i rami di funzioni analitiche di $x$ regolari in un intorno di $x=\infty$ e nulle per $x=\infty$; lo spazio, pure lineare, di cotesti rami di funzione verra indicato con $F$. Indicheremo con $F_{r}$ lo spazio lineare, contenuto in $F$ e quindi in $D$, di quelle serie di potenze $\sum \frac{c_{n}}{x^{n+1}}$ convergenti fuori di un cerchio di centro $x=0$ e di raggio inferiore al numero positivo $r$. Se è $r<s$, lo spazio $F_{r}$ è contenuto in $F_{s}$.

\section{II. - Cenno sulla proprietà delle operazioni distributive, commutabili con $D$ nello spazio $S$.}

7. Un'operazione distributiva $A$ sia univocamente definita per gli elementi $\mathrm{I}, x, x^{2}, \ldots x^{n}, \ldots$ coi quali è costruito lo spazio $S$. Supponiamo inoltre questa operazione commutabile con $D$; sia cioè

$$
A D=D A \text {. }
$$

Ne verrà che $A(\mathrm{I})$ sard una costante; sia $k_{0}$. Volendo $A(x)$, si avrà dalla (7):

$$
A(\mathrm{I})=D A(x), \quad \text { onde } \quad A(x)=k_{\mathrm{o}} x+k_{\mathrm{I}} .
$$

In generale, sard

$$
A\left(x^{n}\right)=K_{n}(x)
$$


dove $K_{n}(x)$ è un polinomio intero della forma

$$
K_{n}(x)=k_{0} x^{n}+n k_{1} x^{n-1}+\left(\begin{array}{l}
n \\
2
\end{array}\right) k_{2} x^{n-2}+\cdots+k_{n}:
$$

ciò si dimostra concludendo da $n$ ad $n+\mathrm{r}$, e fondando il passaggio sulla relazione (7). Il sistema delle costanti $k_{0}, k_{\mathrm{r}}, k_{2}, \ldots$ definisce l'operazione $A$, e i polinomî (8) sono i polinomî di Appell formati con queste costanti.

8. Applicando formalmente l'operazione $A$ ad un serie di potenze si ottiene dapprima

$$
\varphi(x)=c_{0}+c_{1} x+c_{2} x^{2}+\cdots,
$$

$$
A(\varphi)=\sum_{n=0}^{\infty} c_{n} K_{n}(x)
$$

poi, ordinando rispetto alle costanti $k_{n}$, si ottiene per $A$ l'altra espressione

$$
A(\varphi)=\sum_{n=0}^{\infty} \frac{k_{n}}{n !} D^{n} \varphi \text {. }
$$

$\mathrm{Ma}$ questi risultati formali hanno sempre, per le serie $\phi$ di un certo spazio funzionale convenientemente preso entro $S$, una validità effettiva: codesto spazio contiene le funzioni $I, x, x^{2}, \ldots$ e si dirà spazio funzionale di convergenza della ( 10 ). Per dimostrarne l'esistenza, si scelga una successione di numeri positivi decrescenti

tali che la serie :

$$
m_{0}, m_{1}, m_{2}, \ldots \text {, }
$$

$$
\sum_{n=0}^{\infty} m_{n} K_{n}
$$

sia assolutamente convergente; presa allora, nella serie (IO), la funzione $\varphi(x)$ tale che $\left|c_{n}\right|<m_{n}$, le (9) e (Io) risultano assolutamente ed uniformemente convergenti per tutti 1 valori di $x$ di modulo finito. L'insieme delle serie $\varphi(x)$ tali che sia $\left|c_{n}\right|>m_{n}$ appartiene dunque allo spazio funzionale di convergenza (assoluta ed uniforme) della (9).

9. Supponiamo che la funzione $e^{x t}$ appartenga allo spazio di convergenza di $A$. Verrà

Porremo

$$
A\left(e^{x t}\right)=e^{x i}\left(k_{0}+k_{1} t+k_{2} \frac{t^{2}}{\mathrm{I} .2}+\cdots\right) .
$$

$$
k_{0}+k_{\mathrm{I}} t+k_{2} \frac{t^{2}}{\mathrm{I} .2}+\cdots=\alpha(t)
$$


e supporremo questa serie appartenente allo spazio $S_{r}$. La funzione $\alpha(t)$ definisce, mediante i valori

$$
\alpha(0), \quad \alpha^{\prime}(0), \quad \alpha^{\prime \prime}(0), \ldots,
$$

le costanti $k_{\mathrm{o}}, k_{\mathrm{I}}, k_{2}, \ldots$, e quindi l'operazione $A$; daremo a questa funzione il nome di funzione caratteristica dell'operazione stessa.

ı0. Sia ora $\varphi(x)$ appartenente ad $E_{\frac{x}{r}}$. Per il $\S 3$, ciò significa che la serie

$$
\sum n ! c_{n} x^{n}
$$

converge entro un cerchio di centro $x=0$ e di raggio superiore ad $\frac{\mathrm{I}}{r}$, e quindi la funzione

$$
J \varphi=f(t)=\sum_{n=0}^{\infty} \frac{n ! c_{n}}{t^{n+1}}
$$

converge fuori di un cerchio di centro $t=0$ e di raggio $r_{\mathrm{s}}$ inferiore ad $r$. Il prodotto

$$
f(t) \alpha(t)
$$

darà una serie di LAURENT convergente nella corona circolare compresa fra $\mathrm{i}$ cerchi $\left(0, r_{\mathrm{r}}\right)$ e $\left.(\mathrm{o}, r)^{*}\right)$; se $(l)$ è una linea circondante l'origine e tutta contenuta in questa corona, p. es. una circonferenza $\left(o, r^{\prime}\right)$ con $r_{\mathbf{x}}<r^{\prime}<r$, si avrà, come si verifica subito:

$$
\frac{\mathrm{I}}{2 \pi i} \int_{(l)} e^{x t} \alpha(t) f(t) d t=\sum_{n=0}^{\infty} c_{n} K_{n}(x) .
$$

Ma $(\$ \subseteq 4,5)$ la funzione $f(t)$ non è altro che la determinante $J \varphi$ di $\varphi(x)$; inoltre, il secondo membro della (r2) è l'espressione di $A(\varphi)$. Onde per l'operazione $A(\varphi)$, nello spazio funzionale $E_{\frac{I}{t}}$, vale l'espressione in forma d'integrale definito:

$$
A(\varphi)=\frac{\mathrm{I}}{2 \pi i} \int e_{(l)} e^{x t} \alpha(t) J \varphi d t
$$

In particolare, si ha l'espressione delle $K_{n}(x)$ :

$$
K_{n}(x)=\frac{n !}{2 \pi i} \int_{(l)} \frac{e^{x t} \alpha(t) d t}{t^{n+1}}=A\left(x^{n}\right) .
$$

*) Indichiamo con $(a, b)$ il cerchio di centro $a$ e di raggio $b$. 
Si noti, dalla ( I2), che viene

ossia

$$
\varphi(x)=\frac{\mathrm{I}}{2 \pi i} \int_{(l)} e^{x t} f^{\prime}(t) d t
$$

$$
\varphi(x)=\frac{\mathrm{I}}{2 \pi i} \int_{(l)} e^{x t} J \varphi d t .
$$

I I. Siano $A, B$ due operazioni distributive della forma (ro), e siano

$$
\alpha(t)=\sum \frac{k_{n}}{n !} t^{n}, \quad \beta(t)=\sum \frac{b_{n}}{n !} t_{n}
$$

le rispettive funzıoni caratteristiche. Si verifica immediatamente, con calcolo semplicissimo, che l'operazione $A B$ ha come funzione caratteristica il prodotto $\alpha(t) \beta(t)$; ne risulta per quest'operazione, l'espressione

$$
A B(\varphi)=\frac{\mathbf{I}}{2 \pi i} \int_{(l)} e^{x t} \alpha(t) \beta(t) J \varphi d t,
$$

valida nello spazio funzionale $E_{\frac{1}{r}}$ se le serie $\alpha(t), \beta(t)$ appartengono allo spazio $S_{4}$.

Le operazioni $A, B$ sono manifestamente commutabli.

I 2. Fin qui, abbiamo supposto l'operazione $A$ definita mediante il sistema delle costanti $k_{0}, k_{1}, k_{2}, \ldots$, aggiungendo l'iporesi che $e^{\imath t}$ appartenesse, per $|t|<r$, allo spazio di convergenza della serie (ro). Per una simile operazione abbiamo veduto che vale anche l'espressione $(b)$, almeno per le funzion $\varphi$ di uno spazio funzionale conveniente. Ora, questa stessa espressione $(b)$ si può assumere come definizione di operazioni commutabil con $D$, anche in casi in cui, per tal operazioni, non valga una rappresentazione della forma (IO)

Si supponga, infatti, che $\alpha(t)$ sia una funzione analicica uniforme entro una corona circolare di centro o, comprendente nel suo interno la circonferenza $(o, r)$, e sia la funzione $\varphi(t)$ appartenente ad $E_{\frac{1}{\tau}}$. Prendendo come linea $(l)$ d'integrazione la circonferenza $(o, r)$, l'espressione $(b)$ rappresenterà una funzione intera in $x$, e si avrà :

$$
A(\varphi)=\sum_{n=0}^{\infty} g_{n} x^{n}
$$

con

$$
g_{n}=\frac{\mathrm{I}}{2 \pi i n !} \int_{(t)} t^{n} \alpha(t) J \varphi d t
$$


Questa forma dei coefficienti dello sviluppo in serie della funzione $A(\varphi)$ mostra come codesta funzione sia intera, ed appartenga allinsieme $E_{\frac{1}{r}}$. Inoltre, sotto certe condizioni, l'operazione $A$ definita da (b) può essere commutabile con $D$; infatti :

$$
D A(\varphi)=\frac{\mathrm{I}}{2 \pi i} \int_{(l)} e^{x t} \alpha(t) t J \varphi d t ;
$$

ora, per la (6), l'integrale scritto nel secondo membro è uguale a

$$
\frac{\mathrm{I}}{2 \pi i} \int_{(l)} e^{x t} \alpha(t) J D \varphi d t+\frac{\varphi(0)}{2 \pi i} \int_{(l)} e^{x t} \alpha(t) d t:
$$

se ora il secondo di questi integrali è nullo, o se è $\varphi(0)=0$, viene $D A=A D$. Si conclude da ciò che l'espressione $(b)$ può servire a rappresentare operazioni commutabili con $D$, anche quando per codeste operazioni non valga un'espressione in serie di potenze del simbolo $D$, della forma (Io).

\section{III. - Risoluzione dell'equazione (a) per lo spazio $S$.}

I3. Abbiasi ora da risolvere l'equazione

$$
A(\omega)=\varphi,
$$

dove $\varphi$ è una funzione data, appartenente ad $E_{\frac{1}{T}} ; \omega$ è una funzione da determinarsi ; $A$ è un'operazione commutabile con $D$, la cui funzione caratteristica $\alpha(t)$ appartiene ad $S_{r}$.

Entro il cerchio $(o, r)$, la funzione $\frac{\mathbf{I}}{\alpha(t)}$ è regolare, all'infuori di un numero finito di poli. Si può dunque assegnare una corona circolare, di centro $o$, che includa la circonferenza $(0, r)$ ed entro la quale corona il quoziente $\frac{J \varphi}{\alpha(t)}$ sia regolare, ad eccezione di un numero finito di poli. Descriviamo, in questa corona, una linea chiusa $(l)$ circondante l'origine e su cui non si trovi alcuna radice di $\alpha(t)$; l'integrale (che ci dà quindi un'espressione analitica dell'operazione $A^{-\mathrm{r}}$ ):

$$
\omega(x)=\frac{\mathrm{I}}{2 \pi i} \int_{(l)} e^{x t} J \varphi \cdot \frac{d t}{\alpha(t)}
$$

rappresenterà ( $(\mathrm{I} 2)$ una funzione intera, appartenente ad $E_{\frac{I}{r}}$.

Ora, applicando a questa funzione l'operazione $A$, rappresentata da 
verra per il $₫$ II :

$$
A(\omega)=\frac{\mathrm{I}}{2 \pi i} \int_{(l)} e^{x t} \alpha(t) J \omega d t
$$

e, per la (13),

$$
A(\omega)=\frac{\mathrm{T}}{2 \pi i} \int_{(l)} e^{x t} J \phi d t
$$

$$
A(\omega)=\varphi \text {. }
$$

L'equazione $(a)$ è dunque risoluta dalla formula (c), e la soluzione è una funzione appartenente ad $E_{\frac{s}{\tau}}$.

I4. Risolvendo l'equazione (a), si è data contemporaneamente la risposta a varie altre questioni.

a) Dapprima, il problema (a) coincide colla risoluzione di un'equazione differenziale lineare non omogenea, in generale d'ordine infinito:

$$
\sum_{n=0}^{\infty} \frac{k_{n}}{n !} D^{n} \omega=\varphi ;
$$

ciò per la forma (ro) dell'operazione $A$.

b) Nel caso che la serie $\alpha(t)$ sia sempre convergente ed appartenga ad $E_{s}$, la serie

$$
a(t)=\sum_{n=0}^{\infty} \frac{k_{n}}{t^{n+1}}
$$

converge per $|t|>s_{1}, s_{1}<s$. Se $\omega(t)$ è una serie di potenze convergente entro il cerchio $\left(0, s_{2}\right)$ con $s_{2}>s$, la operazione $A$ si può porre sotto la forma

$$
A(\omega)=\frac{\mathrm{I}}{2 \pi \imath} \int_{(l)} a(t-x) \omega(t) d t
$$

valida per $|x|<s_{2}-s_{\mathrm{I}}$ e dove la linea d'integrazione è, p. es., una circonferenza di centro o e di raggio compreso fra $s_{1}$ ed $s_{2}$. La risoluzione dell'equazione $(a)$ equivale pertanto all'inverstone, mediante una serie di potenze di $t$, dell'integrale definto

$$
\int_{(1)} a(t-x) \omega(t) d t=\varphi(x)
$$

c) Sappiamo che se $\omega=\sum g_{n} x^{n}$, si ha $(\$ 8)$

$$
A(\omega)=\sum g_{u} K_{n}(x)
$$

essendo $K_{n}(x)$ i polinomî di Appell relativi al sistema di coefficienti $k_{0}, k_{\mathrm{x}}, \ldots$ Perciò, colla risoluzione dell'equazione $(a)$ è anche risoluto 
il problema di sviluppare la funzione data $\varphi(x)$ in serie ordinata secondo un dato sistema di polinomî di Appell $K_{n}(x)$.

d) Infine la risoluzione della medesima equazione (a) risponde al problema della risoluzione di un sistema di infinite equazioni lineari ad infinite incognite, in cui il determinante dei coefficienti ha la forma:

$$
\left\{\begin{array}{cccccc}
k_{0} & k_{1} & \frac{k_{2}}{2 !} & \frac{k_{3}}{3 !} & \frac{k_{4}}{4 !} & \ldots \\
0 & k_{0} & k_{1} & \frac{k_{2}}{2 !} & \frac{k_{3}}{3 !} & \ldots \\
0 & 0 & k_{0} & k_{1} & \frac{k_{2}}{2 !} & \ldots \\
\ldots & \ldots & \ldots & \ldots & \ldots
\end{array}\right.
$$

I5. La soluzione dell'equazione (a), data dallà formula (c), non è in generale unica. Se infatti $(l),\left(l_{1}\right)$ sono due linee chiuse nelle condizioni indicate al $\int \mathrm{I} 3$, non intersecantisi fra di loro, nell'area chiusa da queste linee si potranno trovare radici di $a(l)$, in numero finito; siano esse

L'integrazione di

$$
\begin{gathered}
a_{\mathrm{r}}, \quad a_{2}, \ldots a_{p} . \\
\frac{e^{x t} J \varphi}{2 \pi i \alpha(t)}
\end{gathered}
$$

eseguita lungo $(l)$ ed $\left(l_{\mathrm{s}}\right)$ darà due funzioni $\omega(x), \omega_{\mathrm{s}}(x)$, la cui differenza sarà espressa da

$$
\omega(x)-\omega_{\mathrm{I}}(x)=\frac{\mathrm{I}}{2 \pi i} \sum_{j=1}^{p} \int_{\left(a_{j}\right)} \frac{e^{x t} J \varphi d t}{\alpha(t)} ;
$$

nel caso che le radici di $\alpha(t)$ siano semplici ${ }^{*}$ ), viene dunque:

$$
\omega(x)-\omega_{2}(x)=\sum_{j=1}^{p} C_{j} e^{x a},
$$

dove le $C$, sono costanti. E d'altra parte evidente che il secondo membro della (I8) soddisfa all'equazione $A(\omega)=0$.

Dall'osservazione precedente, si deduce che i quattro problemi enumerati al $\ \mathrm{I}_{4}$ sono suscettibili di più soluzioni nello spazıo $S$; le differenze di due soluzioni dànno, nello spazio medesimo, la risposta ai seguenti problemi :

*) Si lascia al lettore la facile modificazione che avviene nel caso di radici multiple. 
a) Risoluzione di un'equazione differenziale lineare ad infiniti termini e a coefficienti costanti;

b) Soluzione di un'equazione funzionale della forma:

$$
\int_{(l)} \boldsymbol{a}(t-x) \omega(t) d t=0
$$

c) Sviluppi dello zero in serie ordinate per i polinomî di Appell di un dato sistema;

d) Risoluzione del sistema di equazioni differenziali lineari omogenee in numero infinito e ad infinite incognite, aventi per matrice dei coefficienti 1 l sistema (I 7 ).

16. Consideriamo il caso che $\alpha(t)$ sia una funzione intera. La sua inversa $\frac{I}{\alpha(t)}$ sarà uniforme e in ogni area finita del piano $t$ avrà, per sole singolarità, poli in numero finito. Se la liner d'integrazione $(l)$ include il cerchio $(0, r)$, e se

appartiene ad $E_{\frac{I}{r}}$, la

$$
\varphi(x)=\sum c_{n} x^{n}
$$

$$
A^{-1}(\varphi)=\frac{\mathbf{I}}{2 \pi i} \int_{(l)} e^{x t} \frac{J \varphi}{\alpha(t)} d t
$$

si ridurrt ad uno sviluppo in serie $i$ cui termini avranno la forma

$$
\frac{n !}{2 \pi i} \int \frac{e^{x t} d t}{t^{n+1} \alpha(t)}
$$

Ora, supponiamo dapprima che entro $(l)$ non cada alcuna radice di $\alpha(t)$. La (Ig) è allora il polinomio di Appell $\lambda_{n}(x)$ formato coi coefficienti dello sviluppo in serie

$$
\frac{\mathbf{I}}{\alpha(t)}=l_{0}+l_{1} t+l_{2} t^{2}+\cdots ;
$$

esso soddisfa alla equazione $A\left(\lambda_{n}\right)=x^{n}$, ed appartiene ad un sistema che si dice inverso del sistema $K_{n}(x)$. Si ha cosi il seguente risultato:

"Se $\varphi(x)$ è una funzione appartenente ad $E_{\frac{I}{r}}$, dove $r$ è il minimo «modulo delle radici di $\alpha(t)$, la soluzione dell'equazione $(a)$ è data "dalla serie

$$
\left.\omega(x)=\sum c_{n} \lambda_{n}(x)\right) .
$$

Supponiamo invece che entro $(l)$ vi siano $p$ radici di $\alpha(t)$, e siano 
le radici semplici $a_{1}, a_{2}, \ldots a_{p}$. L'integrale (I9) si ridurrà allora alla forma

$$
\varpi_{n}(x)=\lambda_{n}(x)-\sum_{j=1}^{p} C_{n j} e^{a} j^{x} ;
$$

questo soddisfa, come $\cdot \lambda_{n}(x)$, all'equazione

$$
A(\omega)=x^{n} \text {. }
$$

Questa equazione si può anche soddisfare prendendo in (19) una linea d'integrazione $\left(l_{n}\right)$ variabile con $n$; il numero delle radici di $\alpha(t)$ incluse in $\left(l_{n}\right)$ varierà con $n$, e la $(20)$ si potrà scrivere

$$
\varpi_{n}(x)=\lambda_{n}(x)-\sum_{j=1}^{p_{n}} C_{n i} e^{a_{j} x} .
$$

I7. Un caso interessante, nel quale l'integrazione della equazione (a) si può estendere oltre lo spazio $E_{r}$, è quello in cui sia possibile di assegnare un sistema di costanti $C_{n}$, tale che, essendo $m, g$ ed $b$ numeri positivi, si abbia per $|x|<g$ :

$$
\left|\varpi_{n}(x)\right|<m b^{n} \text {. }
$$

Se allora la serie $\varphi(x)=\sum c_{n} x^{n}$ è convergente in un cerchio di raggio superiore ad $b$, la serie

$$
\omega(x)=\sum c_{n} \varpi_{n}(x)
$$

sard̀ convergente assolutamente ed uniformemente per $|x|<g$, e darà la soluzione dell'equazione $(a)$ che sarà così risoluta per tutto lo spazio $S_{h}$. Questo caso è quello che si presenta nella risoluzione dell'equazione

$$
\omega(x+1)-\omega(x)=\varphi(x),
$$

come si vede dalla bella soluzione che per questa equazione è stata data dall'Hurwitz ${ }^{*}$ ) per il caso di una funzione intera $\varphi(x)$; si vede poi facilmente che la medesima soluzione si può anche estendere al caso in cui $\varphi(x)$ è una serie di potenze convergente in un cerchio di raggio superiore ad $\frac{\mathbf{I}}{2 \pi e}$.

*) Acta Mathematica, t. XX, p. 285 . 


\section{IV. - Cenno sulle proprietà delle operazioni distributive commutabili con $D$, nello spazio $D$.}

18. Riprendiamo l'accenno alle proprietà generali delle operazioni $A$ commutabili con $D$, già indicato nei $\$ \$ 7$ - I $2 ; \mathrm{ma}$ a differenza di quei $\$ \$$, consideriamo la funzione, cui l'operazione $A$ va applicata, come appartenente allo spazio definito al $₫ 6$ e indicato con $D$ : allo spazio cioè delle funzioni determinanti.

Supponiamo dapprima l'operazione rappresentabile mediante uno sviluppo della forma (Io), che scriveremo ora

$$
A(\boldsymbol{f})=\sum_{n=0}^{\infty} \frac{(-\mathrm{I})^{n} k_{n}}{n !} D^{n} f
$$

la serie

$$
\boldsymbol{\alpha}(t)=\sum_{0}^{\infty} \frac{k_{n}}{t^{n+1}}
$$

appartenga ad $F_{r}$. Se allora $f$ è pure un elemento di $F_{r}$, alla $\left(\mathrm{ro}^{\prime}\right)$ si potrà, per il teorema di CAUCHY, dare la forma:

$$
A(f)=\frac{\mathbf{I}}{2 \pi i} \int_{(l)} a(x-t) f(t) d t,
$$

essendo $(l)$ una linea chiusa semplice, circondante il punto $t=0$, e tutta compresa fuori del cerchio $(o, r)$. Il punto $x$ sard esterno a questa linea, ad una distanza dal contorno superiore ad $r$. Ora per questa linea $(l)$ si può prendere anche il contorno composto del segmento rettilineo unente i punti

$$
k+i v, \quad k-i v, \quad(k>r)
$$

e della semicirconferenza avente questo segmento per diametro e rivolta dalla parte negativa dell'asse reale.

Passando al limite per $v=\infty$, l'integrale esteso alla semi-circonferenza tende a zero, e viene cosi per $A(f)$ l'espressione

$$
A(f)=\frac{\mathrm{I}}{2 \pi i} \int_{k-i \infty}^{k+\imath \infty} a(x-t) f(t) d t
$$

con $k>r, R(x)>k+r$.

I9. L'espressione (d) è più generale della ( $\left.5^{\prime}\right)$. Infatti, mentre questa è definita per gli elementi di $F_{r}$, la $(d)$ viene ad avere signifi- 
cato anche per gli elementi di $D$ non appartenenti ad $F$. Se $f$ è definito per $R(x)>b$, la $(d)$ vale per $R(x)>k+b$.

Questa espressione si può ancora modificare. Si ha, per $R(x)>R(t)$ :

onde

$$
\frac{\mathrm{I}}{x-t}=\int_{0}^{\infty} e^{(t-x) u} d u,
$$

Posto quindi

$$
\frac{n !}{(x-t)^{n+1}}=\int_{0}^{\infty} e^{(t-x) u} u^{n} d u \text {. }
$$

$$
\alpha(u)=\sum_{n=0}^{\infty} \frac{k_{n} u^{n}}{n !}
$$

la (d) viene a scriversi :

$$
A(f)=\frac{\mathrm{I}}{2 \pi i} \int_{k-i \infty}^{k+\infty} f(t) \int_{0}^{\infty} \alpha(u) e^{(t-x) u} d u d t
$$

o, invertendo le integrazioni, il che è evidentemente lecito in seguito alle ipotesi fatte :

$$
A(f)=\frac{I}{2 \pi i} \int_{0}^{\infty} \alpha(u) e^{-u x} \int_{k-i \infty}^{k+2 \infty} e^{t u} f(t) d t d u .
$$

Ma si ha $(\$ \$ 4-5)$ che

$$
\frac{I}{2 \pi i} \int_{k-i \infty}^{k+\infty} e^{t u} f(t) d t=G f
$$

onde la (22) ci dà, per l'operazione $A$, l'espressione

$$
A(f)=\int_{0}^{\infty} e^{-u x} \alpha(u) G f d u \text {. }
$$

20. Alle precedenti espressioni ( $\left(\mathrm{O}^{\prime}\right),\left(\mathrm{I} 5^{\prime}\right)$ e $(d)$ della $A$ si è dunque aggiunta anche la (e). Ora, questa nuova espressione offre maggiore generalità delle precedenti in quanto che, in essa, si possono fare ipotesi più larghe sulla natura della $\alpha(u)$, che diremo funzione caratteristica di $A$. Infatti, non sarà pì̀ necessario di supporre, come al $₫$ precedente, che $\alpha$ (u) sia una funzione intera di $u$ data dalla (2I). Basta supporre che $\alpha(u)$ sia una funzione della variabile $u$ data per tutti $i$ valori reali e positivi di questa variabile, insieme alle sue derivate di tutti gli ordini, e tale che, per $x=x_{0}, e^{-u x} \alpha(u)$ sia integrabile fra o ed $\infty$. Lo stesso accadrà allora per tutti i valori di $x$ tali che sia $R(x)>R\left(x_{0}\right)$; e se $f$ è un elemento di $D$, valido per $R(x)>R\left(x_{x}\right)$, il procedimento stesso del $\$ precedente mostra che per $R(x)>R\left(x_{\mathrm{o}}+x_{\mathrm{v}}\right)$, la (e) ha significato e rappresenta una funzione determinante. 
L'espressione (e), sotto questa ipotesi, rappresenta dunque un'operazione applicabile allo spazio $D$, e questa operazione contiene, come casi particolari, quelle della forma (Io'), (I $\left.5^{\prime}\right)$ e $(d)$.

Ora è facile vedere che essa è commutabile colla derivazione; basta infatti notare che

$$
D A(f)=-\int_{0}^{\infty} e^{-u x} \alpha(u) u G f d u,
$$

ma dalla formula $\left(s^{\prime}\right)$ del $\ s$, risulta subito che

onde $D A=A D$.

$$
u G f=-G D f \text {, }
$$

21. Quando la funzione $\alpha(u)$, caratteristica dell'operazione $A(f)$, si specializzi alquanto, è possibile di variare, entro certi limiti, la linea d'integrazione della (e) stessa. Sia infatti $\alpha$ (u) funzione analitica regolare della variabile $u=r e^{2 g}$, per tutti i valori di $r$ compresi fra o ed $\infty$, e per tutti $\mathrm{i}$ valori dell'argomento $g$ compresi fra o e $g_{0}$; inoltre, esista un valore di $x_{0}$ di $x$ per il quale il prodotto $e^{-\imath u} \alpha(u)$ tenda a zero, uniformemente rispetto a $g$, per $r=\infty$. Esisteranno allora infiniti valori di $x$ per i quali quest'ultima proprietà verrà soddisfatta, e saranno tutti quelli interni ad un angolo $g^{\prime}$ di vertice $x_{\circ}$ e coi lati perpendicolari l'uno, all'asse reale, l'altro, alla direzione di argomento $-g_{0}$. Ora, presa convenientemente $f$, si integri la

$$
e^{-\lambda u} \alpha(u) G f
$$

lungo il contorno di un settore circolare formato da due raggi di argomento $0, g_{0}$ e dall'arco intercetto sulla circonferenzd $(o, r)$. L'integrale esteso al contorno sarà nullo; di più, per $r=\infty$, tenderà a zero l'integrale esteso all'arco di cerchio: onde verrà

$$
\int_{0}^{\infty} e^{-x u} \alpha(u) G f d u=\int_{0}^{\infty e^{2} g_{0}} e^{-x u} \alpha(u) G f d u .
$$

L'espressione dell'operazione $A$ è quindi data da un integrale analogo ad $(e)$, ma esteso ad una semi-retta uscente dall'origine e di direzione $g_{0}$. Evidentemente, si può sostituire a questa qualunque semi-retta uscente da $\circ$ e di direzione $g$ compresa fra o e $g_{0}$; la funzione rappresentata da $A(f)$ è espressa, per $x$ preso entro l'angolo $g^{\prime}$, indifferentemente dall'uno o dall'altro membro della (23).

22. Abbiasi una seconda operazione $B$ analoga ad $A$, e di cui $\beta$ (il) 
sia la funzione caratteristica :

$$
B(f)=\int_{0}^{\infty} e^{-x u} \beta(u) G f d u .
$$

Il secondo membro della precedente è, per $R(x)$ abbastanza grande, una funzione determinante di cui

$$
\beta(u) G f
$$

è la generatrice: applicandole l'operazione $A$, verrà dunque

$$
A B(f)=\int_{0}^{\infty} e^{-x u} \alpha(u) \beta(u) G f d u .
$$

Questa relazione mostra che il prodotto di due operazioni $A, B$ del tipo (e) è un'operazione del medesimo tipo, e che la funzione caratteristica del prodotto $\dot{e}$ il prodotto delle funzioni caratteristiche $\alpha(u), \beta(u)$, di $A$ e $B$. $\mathrm{Ne}$ segue ancora che le operazion del tipo (e) sono fra loro commutabili.

23. Ponendo

$$
G f=\varphi(u),
$$

l'espressione (e) dell'operazione $A$ diviene simmetrica in $\alpha(u), \varphi(u)$. Posto $\alpha(u)=G a$, la $A$ si può dunque scrivere:

$$
A(f)=\int_{0}^{\infty} e^{-x u} \alpha(u) G \boldsymbol{f} d u=\int_{0}^{\infty} e^{-x u} \varphi(u) G \boldsymbol{a} d u .
$$

Questa relazione pone in evidenza una legge di reciprocità, che si può formulare dicendo che l'operazione avente come funzione caratteristica $\alpha$, applicata ad $f$, coincide coll'operazione avente per funzione caratteristica $G f$, applicata ad $J \alpha$.

\section{V. - Risoluzione dell'equazione (a) per lo spazio $D$.}

24 Abbiasi da risolvere l'equazione

$$
A(g)=f
$$

dove $f$ è una funzione data in $D ; \mathscr{g}$ è una funzione da determinarsi nel medesimo spazio; $A$ è un'operazione commutabile con $D$, rappresentata da un'espressione della forma $(e)$ ed avente $\alpha(u)$ come funzione caratteristica.

La formula (25) permette di dare immediatamente la soluzione formale del problema. Se infatti s'indica con $B$ l'operazione avente per 
funzione caratteristica $\frac{I}{\alpha(u)}$, viene immediatamente, dalla (25):

e quindi, per il $\subseteq 4$ :

$$
A B(f)=\int_{0}^{\infty} e^{-x x} G f d u
$$

onde :

$$
A B(f)=f
$$

$$
\boldsymbol{g}=\int_{0}^{\infty} \frac{e^{-x u}}{\alpha(u)} G f d u .
$$

L'operazione rappresentata dal secondo membro della (27) è dunque l'espressione formale di $A^{-1}$.

Ora, affinchè questa soluzione formale sia effettiva, basta che la (27) abbia significato per un valore $x_{1}$ di $x$. Se allora $f$ si prende regolare per $R(x)>R\left(x_{2}\right)$, la $(27)$ varrà $(\$ 20)$ per $R(x)>R\left(x_{1}+x_{2}\right)$.

Supponendo $\alpha(u)$, come al $\ 2 \mathrm{I}$, regolare entro un angolo, o più particolarmente ancora, supponeidola intera come al $\$$ I9, basterà, per la risoluzione dell'equazione $(a)$, che esista una semi-retta uscente dall'onigine e di argomento $g$, lungo la quale, per un valore $k=k_{1}$, l'integrale

$$
\int_{0}^{\infty e^{2} g} \frac{e^{-k u}}{\alpha(u)} G f d u
$$

abbıa significato, perchè l'equazione $(a)$ sia risolubile in tutto un angolo del piano $k$, facilmente assegnabile.

25. La risoluzione dell'equazione $(a)$ dà, come al $\$$ I 4 , la soluzione di problemi funzionalı ad essa equivalenti; essi sono $\mathrm{i}$ seguentr:

a) La funzione $g$ del $\$$ precedente risolve il problema di inversione d'integrale definito espresso dall'equazione

$$
\frac{\mathrm{I}}{2 \pi i} \int_{k-2 \infty}^{k+2 \infty} \boldsymbol{a}(x-t) g(t) d t=f(x) .
$$

b) Nel caso che ad $A$ si possa dare la forma ( $\left.10^{\prime}\right)$, croè se la serie $\boldsymbol{a}(t)=\sum \frac{k_{n}}{t^{n+1}}$ è convergente o anche semplicemente sommabile (sommazione esponenziale del Borel), la $\%$ è l'integrale dell'equazione differenziale lineare a coefficienti costanti e non omogenea

$$
\sum(-\mathrm{I})^{n} \frac{k_{n}}{n !} D^{n} \boldsymbol{g}=f
$$

in cui il secondo membro appartiene a $D$. 
c) Per la legge di reciprocità enunciata al $\$ 23$, se la $g(t)$ è sviluppabile nella serie (convergente o anche soltanto sommabile)

la $A(g)$ si può scrivere

$$
g(t)=\sum \frac{c_{n}}{t^{n+1}}
$$

$$
\sum \frac{(-\mathbf{I})^{n} c_{n}}{n !} D^{n} a
$$

Onde l'equazione (a) si può interpetrare come esprimente il seguente problema : « determinare i coefficienti dello sviluppo della funzione $f$ in " serie ordinata secondo le successive derivate della funzione $\boldsymbol{a}$ data "; e la formula (27) ne dà la soluzione.

26. La funzione $\alpha(u)$ si trovi nelle condizioni ammesse al $\ 2$ I per tutti $\mathrm{i}$ valori di $u$ compresi entro un angolo di vertice $u=0$ ed $i$ cui lati abbiano per argomento $g=0$ e $g=g_{0}$. Entro quest'angolo, la $\alpha(u)$ abbia un numero finito di radici semplici $a_{1}, a_{2}, \ldots a_{p}$. All'infuori di questi punti $a_{j}$, siano soddisfatte le medesime condizioni anche per $\frac{\mathrm{I}}{\alpha(u)}$.

In tali ipotesi, gl'integrali

$$
\boldsymbol{g}(x)=\int_{0}^{\infty} \frac{e^{-u x}}{\alpha(u)} \text { Gf } d u, \quad g_{1}(x)=\int_{0}^{\infty e^{2 g_{0}}} \frac{e^{-u x}}{\alpha(u)} G f d u
$$

daranno entrambi, per $\mathrm{i}$ valori di $x$ presi entro un angolo opportuno, soluzioni dell'equazione (a). L'integrazione estesa al contorno di un settore circolare quale è indicato al $\S 2 I$ dà, mediante il teorema di CaUchy,

$$
\mathscr{Y}(x)-\mathscr{Y}_{\mathrm{I}}(x)=\sum_{j=1}^{p} c_{j} e^{-x a_{j}},
$$

essendo le $c$, costanti (nel caso che le $a_{j}$ siano radici semplici; si ommette la nota ed ovvia modificazione del caso in cui le $a$, siano radici multiple).

Con ciò è veduta la plurivocità dell'operazione $A^{-1}$, e si lascia al lettore di enunciare quei problemi, analoghi a quelli enumerati al $\$ 25$, i quali ammettono come soluzione la differenza di due soluzioni della $(a)$.

27. Sia ancora data un'espressione

$$
\boldsymbol{a}(x)=\int_{0}^{\infty} e^{-x u} \alpha(u) d u
$$

rappresentante una funzione determinante per $R(x)>R\left(x_{0}\right)$. 
Si suppone che per $\alpha(u)$ e per tutte le sue derivate, definite fra $u=o$ ed $u=\infty$, siano integrabili le

$$
e^{-x u} \alpha^{(n)}(u) \quad(n=0,1,2, \ldots)
$$

per $R(x)>R\left(x_{0}\right)$. L'espressione (I), come tutte le analoghe in cui al posto di $\alpha(u)$ figurano le sue derivate, tende a zero per $R(x)=+\infty$, come è facile vedere. Ne viene, con successive integrazioni per parti, e posto $\alpha(0)=k_{0}, \alpha^{\prime}(0)=k_{1}, \ldots, \alpha^{(n)}(0)=k_{n}$, che il prodotto

$$
x^{n+1}\left[\alpha(x)-\frac{k_{0}}{x}-\frac{k_{1}}{x^{2}}-\cdots-\frac{k_{n}}{x^{n+1}}\right]
$$

tende a zero per $x=\infty$ nel senso dell'asse reale positivo; in altri termini, $\alpha(x)$ ammette lo sviluppo assintotico, nel senso del PoINCaré:

$$
\frac{k_{\mathrm{o}}}{x}+\frac{k_{\mathrm{x}}}{x^{3}}+\cdots+\frac{k_{n}}{x^{n+1}}+\cdots
$$

Ciò premesso, si consideri un'operazione $A$ della forma (e), e la cui funzione caratteristica $\alpha(l l)$ abbia le proprietà indicate. Presa $f$ in $D$, tale che $G f=\varphi(u)$ abbia la stessa proprietà per $R(x)>R\left(x_{\mathrm{r}}\right)$, e posto

$$
\varphi(0)=c_{0}, \quad \varphi^{\prime}(0)=c_{1}, \ldots, \varphi^{(n)}(0)=c_{n}, \ldots,
$$

il risultato dell'operazione $A\left(f^{\prime}\right)$ sarà una funzıone determinante, regolare per $R(x)>R\left(x_{0}+x_{\mathrm{r}}\right)$, e per la quale varrà lo sviluppo assintotico: (29) $\sum_{n=0}^{\infty}\left[k_{0} c_{n}+n k_{\mathrm{I}} c_{n-1}+\left(\begin{array}{l}n \\ 2\end{array}\right) k_{2} c_{n-2}+\cdots+k_{n} c_{0}\right] \frac{\mathrm{I}}{x^{n+1}}$.

Naturalmente, questo sviluppo, anzicchè assintotico, sard̀ effettivo qualora $\boldsymbol{f}$ ed $\boldsymbol{a}$ appartengano ad $F$. L'espressione precedente di $A(f)$ pone in evidenza la legge di reciprocità enunciata al $\$ 23$.

28. Per terminare, accenniamo brevemente ad alcuni casi particolari dell'equazione (a).

a) L'equazione differenziale lineare a coefficienti costanti e dell'ordine $m$, il cui secondo membro è elemento di $D$,

$$
\sum_{n=0}^{m}(-\mathrm{I})^{n} k_{n} D^{n} \boldsymbol{g}=\boldsymbol{f}
$$

rientra come caso speciale nel problema che abbiamo risoluto. Per la nota scomposizione del primo membro della (30) in un prodotto di fattori operativi semplici, la sua risoluzione si riconduce alla soluzione 
successiva di equazioni della forma

$$
a \boldsymbol{g}-D \boldsymbol{g}=\boldsymbol{f}
$$

dove $a$ è una costante. $\mathrm{La}(3 \mathrm{I})$ si integra immediatamente mediante la formula (27), che nel nostro caso dà :

$$
g(x)=\int_{0}^{\infty} \frac{e^{-x u}}{a-u} G f d u .
$$

L'integrazione si può fare lungo qualsiasi semi-retta uscente dall'origine, di argomento $g$ diverso dall'argomento di $a$, e l'espressione (32) è regolare in $x$ in tutto un semi-piano limitato da una perpendicolare alla direzione $-g$. Se, facendo variare $g$, esso oltrepassa l'argomento di $a$, si troverà una seconda soluzione $g_{1}(x)$ differente dalla prima per una funzione esponenziale

dove $c$ è una costante.

$$
c e^{a x}
$$

Se $\boldsymbol{f}(x)$ appartiene ad $F$, ed è regolare per $|x|>r$, la $\boldsymbol{g}(x)$ sard̀ regolare nel medesimo campo $|x|>r$ all'infuori di un taglio $\tau$ che si estende all'infinito.

Se $f(x)$ ammette una funzione generatrice $G f=\varphi(i)$ finita, insieme a tutte le sue derivate, per $u=0$, ed è $\varphi^{(n)}(u)=c_{n}$, si avrà per l'integrale di (3I) lo sviluppo assintotico

$$
\text { (33) } \sum_{n=0}^{\infty}\left[c_{n} a^{n}+n c_{n-1} a^{n-1}+n(n-1) c_{n-2} a^{n-2}+\cdots+n ! c_{0}\right) \frac{\mathrm{I}}{x^{n+1} a^{n+1}}
$$

e se $f$ appartiene ad $F$, questo sviluppo sarà valido in tutte le direzioni, una sola eccettuata.

Per $f=\frac{I}{x}$, la $\mathscr{g}(x)$ si riconduce alla nota trascendente $l$.

b) L'integrazione dell'equazione $\mathrm{d} 1$ prim'ordine alle differenze finite

$$
\mathscr{g}(x+1)-a g(x)=f(x)
$$

si eseguisce pure mediante la (27), e si ha, escluso il caso di $a=\mathrm{I}$ :

$$
\boldsymbol{g}(x)=\int_{0}^{\infty} \frac{e^{-u x}}{e^{-u}-a} G \boldsymbol{f} d u
$$

L'integrazione si può fare anche qui lungo una semi-retta qualunque uscente dall'origine, e che non contenga alcuna delle radici dell'equazione

$$
e^{-u}=a,
$$

radici allineate parallelamente all'asse immaginario. La direzione dell'asse immaginario è quindi naturalmente esclusa. 
Se $G f=\varphi(u)$, con $\varphi^{(n)}(0)=c_{n}$, varrà per $\boldsymbol{g}$ uno sviluppo assintotico della forma (29). In questo, i coefficienti $k_{n}$ sono quelli dello sviluppo di $\left(e^{-t}-a\right)^{-1}$ in serie di potenze di $u$

e sono dati da

$$
\frac{\mathrm{I}}{e^{-n}-a}=\sum \frac{k_{n} u^{n}}{n !}
$$

$$
k_{\mathrm{o}}=\frac{\mathrm{I}}{\mathrm{I}-a}, \quad(k-\mathrm{I})_{n}=a k_{n},
$$

dove la relazione ricorrente si ottiene sviluppando $(k-I)^{n}$ secondo la regola del binomio, e sostituendo poi gli esponenti mediante indici.

Per il caso di $a=\mathrm{I}$, la (35) non vale; ma poichè $e^{-u}-\mathrm{I}$ contiene $u$ come radice semplice, si potrà, in forza delle relazioni del $₫ 5$, sostituire alla (35) la

$$
\boldsymbol{g}(x)=D^{-\mathrm{x}} \int_{0}^{\infty} \frac{e^{-x u} u}{e^{-t}-\mathrm{I}} G f d u .
$$

In questo caso, le relazioni (36) si riducono per $n=1,2, \ldots$, a quelle dei numeri di Bernould, e la (37), per $f^{*}=\frac{I}{x}$, dà la nota serie assintotica di Strrling.

c) Come ultimo esempio, si potrebbe considerare l'equazione funzionale più generale della $(34)$ :

$$
\sum_{j=1}^{p} b_{j} g\left(x+a_{j}\right)=f(x) ;
$$

ma per lo sviluppo della sua soluzione rimando ad un mio lavoro di parecchi ann fa, pubblicato nel tomo IV della serie IX delle Memorie della R. Accademia delle Scienze di Bologna.

Bologna, 2 maggio I904.

S. PINCHERLE. 